\title{
Carnets
}

Revue électronique d'études françaises de l'APEF

Première Série - 4 | 2012

(Res)sources de l'extravagance

\section{Extravagances de Baudelaire}

\section{Béryl Schlossman}

\section{(2) OpenEdition \\ Journals}

\section{Edição electrónica}

URL: http://journals.openedition.org/carnets/6690

DOI: 10.4000/carnets. 6690

ISSN: 1646-7698

\section{Editora}

APEF

Edição impressa

Data de publição: 1 Janeiro 2012

Paginação: 85-96

Refêrencia eletrónica

Béryl Schlossman, «Extravagances de Baudelaire », Carnets [Online], Première Série - 4 | 2012, posto online no dia 20 junho 2018, consultado o 19 abril 2019. URL : http://journals.openedition.org/ carnets/6690; DOI : 10.4000/carnets.6690

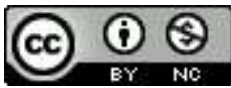

Carnets est mis à disposition selon les termes de la licence Creative Commons - Atribution - Pas d'utilisation commerciale 4.0 International. 


\title{
EXTRAVAGANCES DE BAUDELAIRE
}

BERYL SCHLOSSMAN

Northeastern University

b.schlossman@neu.edu

\begin{abstract}
Résumé
La folie, le caprice, le bizarre et l'excès sont des types d'extravagance depuis la Renaissance et le baroque; si le terme est devenu moins fort depuis, Charles Baudelaire le fait revivre dans plusieurs textes poétiques, surtout des poèmes en prose. On sent, chez Baudelaire, la nuance étymologique de l'errance. L'extravagance entre dans l'esthétique du Spleen de Paris, où on la retrouve dans les arts comme dans la vocation de l'artiste. Dans cet essai, je propose d'explorer le domaine de l'extravagance poétique qui prend forme dans les images, dans les personnages et dans la mise en scène de l'esthétique dans plusieurs poèmes en prose.
\end{abstract}

\begin{abstract}
Madness, folly, the bizarre and excess are types of extravagance formulated in the Renaissance and the Baroque. While the notion of extravagance fades away after those periods, the poet Charles Baudelaire revives it in several poetic texts, especially in prose poetry. The etymological resonance of wandering makes itself felt in Baudelaire's writing. Extravagance enters the aesthetics of Paris Spleen, particularly in the portrayal of the arts and in the artist's vocation. This essay proposes to explore the poetics of extravagance that takes shape in fictional characters, poetic images, and the dramatizing of aesthetics in several prose poems.
\end{abstract}

Mots-clés: Charles Baudelaire, poèmes en prose, esthétique, poétique de la ville Keywords: Charles Baudelaire, prose poetry, aesthetics, poetics of the city 
L'aventure de l'extravagant commence littéralement hors la loi, mais dans l'écriture. Après un début discret du côté du droit canon, où elle indique le détour, le fait de sortir de la voie, l'extravagance - du latin extra-vagare - arrive dans la littérature et les arts. La notion de digression et d'autres dérivés de l'extravagant, comme l'excès, se trouvent à l'usage chez Montaigne. L'essor étymologique du terme s'arrête avant la fin du dix-septième siècle, ses dérivés disparaissent sans trace. Dans l'usage actuel, on ne retient qu'une version fanée de la dépense banale, sans l'émotion amoureuse, sans les effets d'une aventureuse errance ni d'une performance littéraire et philosophique. Or la folie, le bizarre, la notion de divaguer et l'éloignement de la raison semblent tous remonter conceptuellement à l'extravagant, qui reste emblématique de la culture de la Renaissance et du baroque. De nos jours, l'extravagant garde sa résonance imagée uniquement dans la langue populaire des îles britanniques. On relève le sens fort du terme dans le Nord de l'Angleterre, en Irlande et surtout en Ecosse, où la langue parlée donne le verbe "stravage" (errer sans but, vagabonder). On retrouve, chez Baudelaire, la nuance étymologique de cette errance dans des poèmes d'amour dans les "Tableaux Parisiens" et dans des poèmes en prose qui évoquent des figures d'étranger, d'expulsé, de bohémiens, de voyageurs sans repos et des artistes aux abois. L'extravagance se mêle de l'amour, de l'esthétique et surtout de l'errance dans Le Spleen de Paris, où on la retrouve dans la vocation de l'artiste.

L'extravagance habite le sujet baudelairien, qui n'est pas la personne du poète écrivain, mais une voix ou une fiction, chaque fois qu'il se présente - en artiste ou comédien, en observateur solitaire, en voyageur, en amant et parfois en Narrateur. L'extravagance passe par ses paroles, ses gestes et ses envies. La folie, le caprice, le bizarre, l'excès en sont des types repérés du comportement dit extravagant d'après le vocabulaire ancien. Baudelaire l'assimile à sa poétique du surnaturel. Le terme avait l'air suranné depuis le dixhuitième siècle, mais il le fait revivre.

En ville, l'extravagant suit le programme poétique annoncé dans le projet-dédicace célèbre du Spleen de Paris (1: 275-76). L'extravagant flâne ou, tel la prostituée, il fait semblant de flâner, comme s'il ne cherchait pas son objet d'amour ou sa proie au milieu de la foule, ou au jardin public, ou à la barrière. La capitale, c'est la ville vieille et neuve où tout est à vendre. Ce mot à double tranchant fait retour au sujet de la tête du bouffon sublime qui prend un choc mortel - un coup de sifflet, bruit qui rappelle la rue et la circulation - dans "Une Mort Héroïque" (1: 286).

Comme le narrateur du "Cygne", absorbé dans ses pensées lointaines, le poète lyrique erre au cœur de la ville (1: 85-87). II va sur les lieux où la ville se transforme, dans ses petites rues anciennes et sur les grands boulevards modernes, jusqu'à la barrière et dans ses jardins publics, devant les grands parcs rêvés mais interdits. Dans la dédicace provocante du Spleen de Paris, la nouveauté proposée de la poésie en prose prend forme à 
partir de l'errance du poète parmi ses choses parisiennes: "Quel est celui de nous qui n'a pas [...] rêvé le miracle d'une prose poétique musicale sans rythme et sans rime, assez souple et assez heurtée pour s'adapter aux mouvements lyriques de l'âme, aux ondulations de la rêverie, aux soubresauts de la conscience?" (1: 275-76). A la place de la prosodie, Baudelaire propose des images de mouvement pour exprimer l'âme, l'esprit, la pensée et la conscience. La nouveauté esthétique est un art à la fois matériel et invisible, dont l'inspiration à la fois souple (dérivée du chant, du rêve éveillé, et de la forme rythmée des vagues) et heurtée (tout en soubresauts, dérivée des expériences de choc) vient de la vie moderne: "C'est surtout de la fréquentation des villes énormes, c'est du croisement de leurs innombrables rapports que naît cet idéal obsédant" (1:276). A partir de ce projet, l'évocation du vitrier se propose: d'une part, le rêve - l'art, la couleur, le Beau - et d'autre part, la misère d'un quartier pauvre, le coup parti et la jouissance de l'extravagant. L'attaque contre le vitrier est gratuit, et le narrateur ironique ne manque pas d'évoquer le Mal (et des fleurs).

Le cri du vitrier attire l'attention du Narrateur. Le vitrier habillé de sa transparente marchandise devient une allégorie de l'image tout en reflets, de la transparence, de la réflexion et de l'architecture fragile. De sa personne, il évoque l'art. Comme le Poète dans la rue, il trébuche, poussé par le Narrateur. Du carreau on arrive à l'image encadrée, puisqu'à l'époque on voyait des estampes et des tableaux dans les boutiques des vitriers. Le projetdédicace donne l'impression d'un duel verbal avec Houssaye. Sa tentative de 'chanson' citée, "O Vitrier", devient pour Baudelaire le prétexte de son poème en prose "Le Mauvais Vitrier" (1: 285-7, 1309-1311). A partir du cri de la rue, Baudelaire engage le dialogue de poète prosateur de la même manière que son personnage s'attaque au pauvre vitrier: plus le personnage du poète est extravagant, et plus il est fort. Son pot de fleurs est une allusion pointue aux Fleurs du Mal, engin explosif qui ruina son éditeur après le procès perdu par de son auteur. Une fois lancé, le pot de fleurs provoque une destruction éclatante comme d'un palais de cristal. L'explosion est signée par le Narrateur avec le cri de "La Vie en beau!", la réplique au cri de "O! Vitrier" chez Houssaye.

La question de la beauté, la notion de l'extravagance et le tableau de Paris élaborés dans "Le Mauvais Vitrier" transforment les données du cri de la rue en œuvre d'art. Au poème en prose sentimental d'Arsène Houssaye, Baudelaire répond par une sorte d'escrime verbale lorsqu'il insiste sur le goût sadique et le besoin d'excès chez son personnage (et ses terrifiants amis, pourtant de grands timides). II ne serait pas difficile d'imaginer que la lecture du poème de Baudelaire faisait oublier le poème de Houssaye. Inutile de signaler que Houssaye n'a pas satisfait la demande de Baudelaire, qui cherchait un éditeur pour ses petits poèmes en prose.

Les verres colorés demandés par l'extravagant Narrateur du "Mauvais Vitrier" rappellent le poète cabotin, Samuel Cramer, le premier des extravagants nés sous la plume 
de Baudelaire. "Pris d'un caprice bizarre", ce personnage, le poète héroïque de La Fanfarlo, insiste pour voir la femme de ses rêves porter le rouge (1: 577). L'illusion théâtrale, c'est toujours d'abord la vie en Beau, et plus c'est faux, mieux c'est - c'est l'avis du poète, Baudelaire, tout autant que de ses personnages. II transforma la modernité en inventant un nouveau réalisme qui passe par le surnaturel. Les vitres magiques et paradisiaques sont des indices de son ambition esthétique. Rien de tel ne se manifeste dans le poème en prose d'Arsène Houssaye.

Héros solitaire, acteur et comédien dans un drame inconnu, l'écrivain se présente non pas devant un pupitre ou assis dans une bibliothèque, mais engagé physiquement, pris dans le réseau des croisements et en train de marcher. Son drame invisible rappellerait celui de Hamlet, toujours apprécié par les romantiques et au Second Empire, associé à l'escrime et au duel. Baudelaire évoque l'escrime aux mouvements à la fois souples et heurtés, où le fleuret est devenu une langue poétique. L'origine du nom du poète, l'arme qui coupe, se transforme - grâce à l'escrime - en plume provocante. A partir de cette image, celle du saltimbanque va prendre son essor, pour exprimer le parcours solitaire de l'écrivain. L'âme, l'esprit et les sens se retrouvent dans l'image de l'artiste qui travaille comme un artisan, avec son corps. C'est l'escrime sans fleuret, dans le geste du poète errant à travers les rues, coudoyé par les foules.

Maudite et magique, la ville baudelairienne est voilée par l'illusion et la fantasmagorie. Dans le réseau haussmannien où le sujet se promène, la construction allégorique de la Ville est un ensemble de ruines noircies de fumée et d'ébauches précoces. La ville est vieille de sa longue histoire, ses rues sont en train de disparaître dans les travaux ou à peine creusées. Le sujet qui tourne dans ce labyrinthe cherche à renaître par l'Amour, alors que la Mort qui habite la vieille ville guette sa mélancolie à chaque tournant, lui qui porte le souvenir de ses pauvres morts, trop vite passés vers le monde des ombres, la ville sous la ville. Les images des ancêtres, des hôpitaux, des revenants, des tambours funèbres et de la ville souterraine frappent le lecteur des Fleurs du Mal depuis le commencement du recueil, et jusqu'aux "Tableaux Parisiens" qui deviennent de plus en plus explicites concernant ces présences troublantes qui sont plus chez eux que les vivants. Liée à l'extravagance, l'étrangeté chez Baudelaire est à lire dans ce sens tout autant que la notion de l'artiste étranger au monde bourgeois du progrès.

Le personnage extravagant le plus moderne, peut-être, chez Baudelaire, c'est le Poète anonyme qui parle dans "A Une Passante". II descend dans la rue à la recherche d'un amour au milieu de la foule. C'est un drame du regard et de la beauté, sous la menace de la mort:

Moi, je buvais, crispé comme un extravagant, 
Dans son œil, ciel livide où germe l'ouragan,

La douceur qui fascine et le plaisir qui tue.

Le bruit et d'autres formes du choc sont portés jusqu'à lui par la foule. Quasi médium, il représente ou incarne dans son corps ou sa gestualité ce choc, qui se transmet principalement dans la crispation de l'extravagant, dont le regard transforme la femme endeuillée en statue. Le temps d'arrêt se joue de façon imperceptible, mais le climat tropical produit un orage final, une Nuit allégorique au-delà de la vie du Poète (lui aussi allégorique). Le "moi" du poème se révèle finalement comme le creuset intime d'un regard, d'un désir, et d'un silence extatique et mortel. Son cri d'amour à la fin du sonnet en appelle à la beauté, complice d'une vie colorée - mais elle disparaît, blanc sur noir. L'amour est aussi difficile à trouver que la vie en beau dans un quartier pauvre.

"A Une Passante" est un poème d'amour écrit dans un style lyrique, mais l'événement qui s'y produit ne peut fonctionner selon un modèle de rapprochement amoureux ni une expérience auratique de l'amour. II y a un croisement en éclair, ensuite l'abîme s'installe dans le temps, dans l'espace du poème. La distance qui sépare le sujet narrateur de son objet voilé s'étend à l'infini. Le temps des retrouvailles anticipées est abandonné au choc du "Jamais".

L'oxymoron - crispé comme un errant de l'au-delà - nous montre celui qui va trop loin. Le passage est dédoublé, les deux personnages sont des sujets de pétrification. La femme aperçue passe sur sa jambe de statue, alors que l'amant en puissance se déclare crispé et extravagant. La pétrification du désirant s'accompagne de son contraire, du mouvement en dehors de son chemin, de la folie de l'errance et de l'excès de la passion. A la fois figé et fuyant, ce sujet de la Mort ressemble étrangement, au moins par la rhétorique, à la femme en grand deuil qu'il admire.

Ces oxymorons sont pris par la violence de la ville, d'une part, et du désir, de l'autre. L'ensemble est d'une étrangeté et d'un lyrisme qui sont la marque de l'extravagant, acteur dans un théâtre invisible, virtuel. Dans son commentaire, Benjamin fait une analyse saisissante du poème selon le critère non pas de l'amour mais de la sexualité: "Baudelaire führt die Figur der sexuellen Perversion, die ihre Objekte in der Straße sucht, in die Lyrik ein. Das Kennzeichnendste aber ist, daß er das mit der Zeile 'crispé comme un extravagant' in einem seiner vollkommensten Liebesgedichte tut 'A une Passante' [Baudelaire introduit dans la poésie lyrique la figure de la perversion sexuelle qui cherche ses objets dans la rue. Mais ce qui est le plus reconnaissable, c'est qu'il le fait avec le vers 'crispé comme un extravagant' dans un de ses poèmes d'amour les plus achevés" (V.1: J21a, 4).

Dans le vers "Moi, je buvais, crispé comme un extravagant", Benjamin voit l'instance du désir comme un choc. Ce bouleversement violent se donne dans une analogie, dans la 
comparaison "comme", qui laisse l'extravagant dans l'ombre de l'image. Le substantif de l'extravagant est venu du baroque, pour marquer la folie et l'excès d'un cheminement qui transforme le parcours du passant. Benjamin prend le parti de l'extravagance baudelairienne, pour transformer l'acte métaphorique de boire en l'acte de succomber à l'intoxication, à la corruption, à la ruine... ou à quelqu'un. Dans ce vers, le rythme et la ponctuation des trois premières syllabes produisent des effets de staccato et de syncope, marquant la césure à la deuxième virgule. L'hémistiche commençant par "crispé" fait résonner les consonnes occlusives, sibilantes et liquides qui sont comme le nom de la passante anonyme. "Boire dans son œil" dévoile l'instant de l'ivresse érotique dans l'hyperbole du regard. "Je buvais" fait écho aux voyelles de la première rime tout en anticipant la dernière rime du sonnet.

Or la perversion relevée par Benjamin rappelle plutôt la démarche de la prostituée que celle de l'amoureux, ou bien les personnages timides et sadiques en même temps du "Mauvais Vitrier". L'apostrophe de la fin du sonnet est une accusation rancunière tout autant qu'une déclaration d'amour: ce Poète doit quelque chose à Samuel Cramer, déjoué par les deux femmes qu'il croyait avoir séduites.

Baudelaire produit une autre version moderne de l'extravagance dans ses portraits de sujets anonymes, descendus dans la rue à la recherche d'une rencontre, d'une liberté ambiguë, d'une jouissance interdite, d'un amour fugitif - ou bien à la recherche d'un vers poétique, d'une réflexion ou d'une image. Le poète-escrimeur du "Soleil" part à l'aventure d'un vers inconnu, d'une figure cachée:

Je vais m'exercer seul à ma fantasque escrime,

Flairant dans tous les coins les hasards de la rime,

Trébuchant sur les mots comme sur les pavés,

Heurtant parfois des vers depuis longtemps rêvés (1: 83).

Ce Poète anonyme est absorbé dans l'errance passionnée de son travail invisible: le poème le met en scène dans son parcours des croisements imprévus des rues parisiennes. C'est un personnage tout en mouvement, occupé par l'ondulation du vers et de la rime et absorbé par les trébuchements de sa démarche et les chocs de la rue. Ces trébuchements liés aux soubresauts des acrobates - sont à prendre à la lettre et de façon métaphysique, lorsque le poète remplace les pavés par des mots et des souvenirs. Dans sa version de l'escrime, à force de s'élancer et de trébucher, il frôle la chute. Le verbe au participe présent signale le travail poétique et l'aspect matériel et sensuel de son mouvement.

Dans ce poème connu depuis 1857 mais probablement plus ancien, le soleil est évoqué de façon romantique comme père. Ce père est comparé au poète, tandis que la Nature "enchanteresse et rivale" provoque la défaite du poète dans le poème en prose, "Le 
Confiteor de l'Artiste" (1: 278-279). Comme d'autres artistes héroïques de Baudelaire, "Le Soleil" rappelle Hamlet à la voix tragique, comique, amoureuse et poétique. Par son extravagance et sa fragilité, par sa mortelle puissance de comédien devant ses souvenirs et par sa clairvoyance devant la tâche mortelle de vengeance, qui le mènera à l'escrime et à la mort, Hamlet évoqué par Baudelaire incarne le rôle de l'artiste.

Les mêmes éléments sont recomposés dans "Une Mort Héroïque" autour du spectacle sublime donné par Fancioulle, comédien et bouffon, mime et saltimbanque en même temps (1: 285-287). Ce fou appelé "Garçon" (en italien, fanciullo) est un artiste - sans véritable nom propre - qui joue des rôles. En cela il est proche de l'inspiration baudelairienne de l'anonymat, à la fois urbaine et esthétique. Fancioulle, l'artiste chargé d'amuser les grands, est d'abord complice du tyrannique Prince qui ressemble aux rois du Spleen, ces grands sensibles qui agissent avec une cruauté suicidaire. Le Prince apprécie son fou, presqu'un ami. Mais Fancioulle participe à une conspiration contre lui. On n'apprend pas pour quelle raison le fou trahit son Prince. Se laisse-t-il influencer par des conspirateurs habiles, ou bien se révolte-t-il contre la tyrannie? Devient-il conspirateur par jeu, par ennui, par conviction, par rage pure ou par erreur? On ne peut pas savoir. On n'apprendra rien làdessus du Narrateur clairvoyant, celui qui voit l'auréole invisible du comédien exceptionnel.

Dans "Une Mort Héroïque", l'artiste se présente de façon physique et expressive. La syntaxe de la phrase descriptive est heurtée, comme en soubresauts, avec une suite de verbes à l'imparfait. Ensuite, le vocabulaire théologique de la grâce et du mystère est remanié pour les besoins de l'art afin d'indiquer la virtuosité et la souffrance du bouffon:

Ce bouffon allait, venait, riait, pleurait, se convulsait, avec une indestructible auréole autour de la tête, auréole invisible pour tous, mais visible pour moi, et où se mêlait, dans un étrange amalgame, les rayons de l'Art et la gloire du Martyre. Fancioulle introduisait, par je ne sais quelle grâce spéciale, le divin et le surnaturel jusque dans les plus extravagantes bouffonneries (1: 321$)$.

Deux fois dans le poème, l'extravagance de l'art de Fancioulle s'associe à l'étrangeté. Ici c'est l'auréole qui est étrange par le mélange de l'esthétique et du sacré.

Léger et enjoué, l'artiste aux merveilleux talents incarne ses personnages de façon idéale, parfaite. En mouvement perpétuel, il envoûte tous ses spectateurs. Mais le Prince pâlit d'une pâleur nouvelle et brûle d'un feu jaloux tout en applaudissant "ostensiblement les talents de son vieil ami, l'étrange bouffon, qui bouffonnait si bien la mort" (1: 322). Pâle et brûlant, ce Prince aime l'art de Fancioulle, et le bouffon lui-même, qu'il va punir de façon particulière. Le coup du sifflet est une réplique à la conspiration, dans l'intrigue des coulisses, 
qui prolonge la mise en scène hyperbolique, par Fancioulle, de ses bouffonneries extravagantes:

Fancioulle, secoué, réveillé dans son rêve, ferma d'abord les yeux, puis les rouvrit presque aussitôt, démesurément agrandis, ouvrit ensuite la bouche comme pour respirer convulsivement, chancela un peu en avant, un peu en arrière, et puis tomba roide mort sur les planches (1: 322).

Etrange, en effet: la mise en scène passe de façon surnaturelle de l'expression artistique à une pantomime improvisée de la Mort, réelle.

La raillerie médiévale que Baudelaire affectionnait aboutit au néologisme du Narrateur, "bouffonner la mort", qui donne toute leur résonance sanglante d'ironie aux termes de "faveur capitale" et de "bon comédien". Ce poème transforme l'étrangeté en art dans les extravagantes bouffonneries, qui font rêver, qui enchantent, et qui reprennent l'enjeu des Fleurs du Mal pour le situer dans Le Spleen de Paris. Baudelaire prend l'étrangeté au pied de la lettre. Le bizarre, le capricieux, le Beau - voilà un étrange amalgame, une esthétique qui rayonne d'une Auréole invisible.

La Passion esthétique et la souffrance du Martyre forment la double auréole de l'artiste miraculeux. Au-delà de tout le pouvoir de l'effacement de sa punition, il arrive à bouffonner la mort. Un coup de sifflet, ordonné par le Prince, rompt violemment le silence magique de son art, comme dans un rappel à l'ordre ou un défi de spectateur mécontent. Fancioulle va plus loin que le Prince, puisqu'il est capable de transformer la punition en œuvre d'art, la chute en drame. Il jouit, cet extravagant, de la faveur capitale de mourir en beauté, tandis que les autres conspirateurs, témoins de sa mort, sont tout simplement "effacés" de la vie, comme on efface une phrase sur du papier.

Dans le poème en prose, "Le Confiteor de l'Artiste", l'escrimeur est un artiste passionné de Beauté qui crie de frayeur devant le Beau qu'il ne peut pas atteindre (1: 278279). Cet artiste rappelle par son cri le cri de la rue du vitrier bien que le poème déguise cette analogie par l'invocation de la nature. En même temps, son cri ressemble étrangement au coup de sifflet qui rompt le charme du silence dans "Une Mort Héroïque". Le souffle est coupé de façon littérale, c'est une hyperbole qui prend la forme d'une allégorie. A force de bouffonner la mort, Fancioulle va mourir vraiment, mais pourtant sa mort est représentée comme la conclusion de la féerie interrompue. Le réveil meurtrier provoqué par le coup de sifflet remet l'artiste en scène, cette fois-ci en martyr. II serait impossible pour le spectateur de distinguer l'acteur de son personnage dans ce dernier acte. En cela, Fancioulle atteint à la parfaite idéalisation signalée par le Narrateur et liée à l'invisible auréole. 
La Nature, le Beau et L'Infini envoûtent l'artiste. Par son idéal, par son art, par sa passion, il voudrait atteindre à la hauteur de sa tâche. II y a deux interprétations possibles: ou bien tout le texte fournit une confession de l'extravagance de l'art, de son ambition de rendre I'Infini, ou bien, "vaincu" par l'étude, l'artiste se confesse en poussant un cri de frayeur. Cette allégorie de l'idéal de l'art porte sur l'émotion et l'expression dans le contexte du déguisement et de l'anonymat. Contrairement aux écrivains célèbres et autoritaires, l'artiste allégorique présenté dans le poème touche, par le sens de sa défaite, à l'essentiel de son art. La confession de l'artiste a un aspect rituel et liturgique qui occulte l'identité d'une vie biographique ou personnelle: le 'confiteor' ne se confond pas avec une confession romantique mais reste rigoureusement dans le domaine de l'esthétique grâce à la figure allégorique de l'artiste criard. Sans la reconnaissance de l'allégorie, le lecteur peut tomber dans la confusion répandue par la critique depuis la fin du dix-neuvième siècle et jusqu'à nos jours entre la vie personnelle de Baudelaire et l'accomplissement réussi de sa vision esthétique, vision qui a transformé la littérature et les arts modernes.

Le poète transforme les soubresauts des saltimbanques en de brusques accès de conscience, des acrobaties virtuelles et pensées. Lisibles depuis "Le Soleil" et les poèmes en prose, les soubresauts entrent dans les gestes de M. G., le peintre de la vie moderne, "s'escrimant avec son crayon, sa plume, son pinceau, faisant jaillir l'eau du verre au plafond, essuyant sa plume sur sa chemise, pressé, violent, actif, comme s'il craignait que les images ne lui échappent, querelleur quoique seul, et se bousculant lui-même" (2: 693). La vision que présente Baudelaire de M. G. ressemble au travail du saltimbanque par la passion, la rapidité et l'acuité. Le crayon et la plume de M. G. reprennent le travail et la gestualité de l'escrime. De nouveau, la figure de l'escrime indique la rhétorique et l'écriture, plus le jaillissement d'une fontaine, la résurrection et le duel. Baudelaire partage l'art entre la mémoire et l'ivresse ou la fureur (2:698-299).

L'inscription du travail poétique dans les poèmes à résonances autobiographiques ne change rien à l'anonymat et à l'invisibilité de la tâche poétique. La position critique de Baudelaire, qui s'oppose au culte de la personnalité et à la sacralisation du personnage de l'écrivain (dont Victor Hugo est un bon exemple), indique la portée toute moderne de sa propre démarche poétique. Ses figures du Poète anonyme sont à comparer au sujet désirant (et parfois amoureux) qui suit ses objets érotiques dans la rue: la Prostitution, réplique aux mythes de la Flânerie, est à lire dans le même réseau. Le beau et le bonheur font signe de loin, depuis le martyre et le sacrifice, depuis la prostitution et l'art du saltimbanque. Le Poète au travail et le sujet amoureux se réunissent dans l'esthétique, et se retrouvent non seulement dans la rue, mais à la rue.

En ce sens, "A Une Passante" apparaît comme une version poétique, lyrique et tragique, du projet même du Spleen de Paris. Le berger extravagant d'autrefois cède le pas 
devant le poète moderne, pris dans la magie des rencontres et dont la solitude se prête aux gestes du martyr. L'émotion de l'amour et de la sensibilité esthétique situent l'extravagance dans les rues de la ville, dans les rues qui se croisent - depuis la Renaissance jusqu'à la capitale de modernité. Au royaume de la beauté, ces croisements - qui se mettent à l'œuvre dans l'esprit, dans les sens et dans les arts - créent les correspondances: au royaume du choc, de la destruction de l'illusion et de la mort, c'est l'allégorie qui domine. La ville est parfois figurée par une salle de spectacle, comblée de spectateurs, ou bien par une cour royale, remplie d'intrigues, de conspirations, de passions secrètes.

Les trois personnages, les répondants allégoriques du poète selon Jean Starobinski, rappellent surtout les personnages allégoriques et les voix intimes des poèmes du spleen. Le Narrateur est à part: il compose une interprétation poétique, railleuse et amère, de la riposte royale à la conspiration. La mise à mort de Fancioulle par le Prince cruel, jaloux et tyrannique est une fable à trois instances extravagantes - l'art, le désir (et l'amitié), et le pouvoir. II n'y a pourtant que deux personnages qui comptent, le Prince et son bouffon. C'est encore un duel où l'artiste est martyrisé. Pourtant ce héros de l'art ne crie pas de frayeur comme l'artiste du "Confiteor de l'Artiste". L'hyperbole - l'intensité dramatique - n'est pas dans la voix, mais passe dans une autre forme d'expression. Saltimbanque autant que poète, Fancioulle réagit et s'exprime dans son corps et dans son souffle, lorsqu'il tombe mort à la suite de son spectacle, qui est d'une maîtrise sublime et d'une beauté inoubliable. L'extravagance, l'hyperbole et la convulsion marquent son art vivant autant que la mise à mort qui l'afflige à distance. Cette mise à mort transforme le bouffon bien-aimé en étrange bouffon. Dans la fiction ou la fable de sa mort héroïque, l'étrangeté et l'accomplissement du grand art de Fancioulle sont emblématiquement dessinés par l'auréole qu'il porte.

Comme les tableaux religieux du Christ et des saints portent une auréole ou une couronne à rayons, les Poètes avec majuscule lisent dans l'invisible et portent une auréole que d'autres ne peuvent pas voir. Dans "Le Vieux Saltimbanque" l'extravagance est un luxe perdu dans la foule. Le silence, la pauvreté et l'attitude figée montrent le vieil artiste à l'écart du bruit et du mouvement de la ville en fête, tandis que le Poète - celui qui le peint et parle pour lui - est clairvoyant comme le Narrateur racontant l'histoire de Fancioulle dans "Une Mort héroïque". En fin de compte, après la dépense ou le sacrifice de l'extravagance, l'illusion de l'art s'évanouit. II ne reste que l'errance, le vide ou l'exil. C'est alors qu'intervient l'imagination. Crispé comme un extravagant, le Poète devant la Passante dans "A Une Passante" ne peut la suivre (1: 92). Par une sorte d'accusation (ou de consolation), il s'imagine l'atteindre dans l'éternité. Baudelaire avait d'abord écrit: "tremblant comme un extravagant", avant de se corriger. Le tremblement d'un témoin ou d'un narrateur ne vaut pas le tableau vivant du choc. II choisit l'immobilité crispé du saltimbanque pour rendre l'effet 
de ce canevas d'amour manqué, renvoyé au tableau noir de l'éternité, qui manque peut-être de conviction.

Les personnages du Narrateur et du Poète dans ces poèmes en vers ou en prose sont des témoins, anonymes eux aussi, tout autant que le poète au soleil, l'artiste au surnom enfantin de Fancioulle ou le vieux saltimbanque. Piégé par le Prince cruel qui sera le premier à le regretter, Fancioulle, artiste aux extravagantes bouffonneries, est crispé par un choc mortel. Le Narrateur, tout tremblant et en larmes, indique la dernière extravagance. Le comédien serait allé jusqu'au bout de sa bouffonnerie en improvisant la mort de son personnage. Fancioulle atteint à la perfection de son art: l'acteur et son personnage sont fondus en un. Au-delà de limites, l'extravagant nous fait entrer dans un réseau de croisements où se côtoient l'amour coloré et le noir destin, la poésie et la marchandise, et enfin, l'art et la prostitution. Ce réseau de croisements est le fond de l'errance réelle, anonyme et déchirante, des déracinés, des saltimbanques et des figures du Poète que Baudelaire - d'un coup de théâtre qui transforma le paysage littéraire de deux siècles, sinon trois - rend à la modernité rude mais sublime de la grande ville. 


\section{Bibliographie}

BAUDELAIRE, Charles (1975 et 1976). CEuvres complètes en deux volumes. Paris: Gallimard.

BENJAMIN, Walter (1974 - 1989). Gesammelte Schriften, sous la direction de Rolf Tiedemann and Hermann Schweppenhäuser. Frankfurt am Main: Suhrkamp.

CHAMBERS, Ross (1971). "L'art sublime du comédien ou le regardant et le regardé". In: Saggi e ricerche di letteratura francese, vol. 11, pp. 191-260.

ChAmBers, Ross (1987). Mélancolie et opposition: les débuts du modernisme en France. Paris: José Corti.

Chambers, Ross (1985). "Baudelaire's Street Poetry". In: Nineteenth Century French Studies, vol. 13, pp. 244-59.

LAUREL, Maria Hermínia Amado (2001). Itinerários da Modernidade: Paris, espaço e tempo da modernidade poética em Charles Baudelaire. Coimbra: MinervaCoimbra

MACCHIA, Giovanni (1985). Le Rovine di Parigi. Milano: Mondadori.

MuRPHY, Steve (1996). "La Scène parisienne: lecture d'Une Mort Hérö̈que de Baudelaire". In: Le Champs littéraire, mélanges offerts à Michael Pakenham. Amsterdam: Rodopi, pp. 49-61.

Schlossman, Beryl (1991). The Orient of Style: Modernist Allegories of Conversion. Durham et Londres: Duke University Press.

SCHLOSSMAN, Beryl (2000). "La Nuit du poète: Baudelaire, Benjamin et la passante." In: Dalhousie French Studies, vol. 53, pp. 12-26.

SCHLOSSMAN, Beryl (2005). "Baudelaire's Place in Literary and Cultural History". In: Rosemary Lloyd, Editor. Cambridge Companion to Baudelaire. Cambridge: Cambridge University Press, pp. 175-185.

Schlossman, Beryl (2008). "Walter Benjamin, The Writer of Modern Life: Essays on Charles Baudelaire". In: H-France Review Vol. 8, numéro 87. <URL: http://www.hfrance.net/vol8reviews/vol8no87schlossman.pdf>

STAROBINSKI, Jean (1967). "Sur quelques répondants allégorique du poète". In: RHLF vol. 67 , pp. 402-412.

StAROBINSKI, Jean (1970). Portrait de l'artiste en saltimbanque. Paris: Flammarion. 